$\geq 18$ years admitted with CDI from 2006-2018 were included. Patients with RA were identified using the International Classification of Diseases (ICD) 9 and 10 codes. Categorical variables between CDI patients with and without RA were compared using Chi-squared test. Temporal trends of hospitalization charges, length of stay (LOS) and inpatient mortality were assessed using the Cochrane Armitage test. Predictors of inpatient mortality were assessed using multivariable logistic regression adjusting for: age $\geq 65$, sex, race, presence of RA, and Elixhauser comorbidity index.

Results: There were 4,396,945 hospitalizations with a discharge diagnosis of $\mathrm{CDI}$ in the study period, of which $111,336(2.5 \%)$ had a coexisting diagnosis of RA. Over the study period, the proportion of CDI patients with a diagnosis of RA increased from $1.7 \%$ in 2006 to $3.3 \%$ in 2018 with a statistically significant trend of rise $(p$ trend $<0.001)$. There were significantly more females in the RA group (78.2\%) than without RA $(57.5 \%), p<0.001$. RA group was significantly younger ( 68.0 vs $70.7, P<0.001)$. Races were similar between the groups.

The inflation-adjusted average charge for hospitalization for CDI without RA was significantly higher than for CDI with $R A$ ( $\$ 95,863$ vs $\$ 73,025, p<0.001)$. There was an increasing trend in inflation-adjusted average charge for hospitalization for both CDI without RA (\$76,966 in 2006 to $\$ 104,552$ in 2018, $\mathrm{p}<0.001$ ) and CDI with RA ( $\$ 55,689$ in 2006 to $\$ 77,295$ in 2018, $p<0.001$ ). Patients with or without RA had the same median length of stay (LOS) of 7 days. But when trended over time, for patients without RA, the median LOS decreased from 8 days in 2006-08 to 6 days in 2016-18 whereas for patients with RA, the median LOS fluctuated between 6 and 7 days over the course of study period.

CDI patients without RA had a significantly higher mortality over the study period than those with RA $(7.8 \%$ vs $6.1 \%, p<0.001)$. Among non-RA CDI patients, mortality decreased from $9.3 \%$ in 2006 to $6.3 \%$ in 2018 ( $p$ trend $<0.001$ ). Hospitalizations of people with CDI and comorbid RA showed an overall decreasing trend ( $p$ trend $<0.001$ ).

The multivariable analyses found age $\geq 65$ was associated with increased inpatient mortality (adjusted odds ratio [aOR], 1.77, 95\% confidence interval [Cl], 1.731.80), whereas a diagnosis of RA was associated with a decreased mortality (aOR $0.86,95 \% \mathrm{Cl}, 0.81-0.91$ ) (Table 1).

Table 1. Predictors of inpatient mortality

\begin{tabular}{|c|c|c|c|c|}
\hline & \multirow[t]{2}{*}{$\mathrm{aOR}$} & \multicolumn{2}{|c|}{$95 \%$ C.I. for aOR } & \multirow[t]{2}{*}{ P-value } \\
\hline & & Lower & Upper & \\
\hline Age $\geq 65$ & 1.765 & 1.730 & 1.801 & $<0.001$ \\
\hline \multicolumn{5}{|l|}{ Hospitalization years } \\
\hline -Years 2006-2011 & Ref & & & \\
\hline -Years 2011-2014 & 0.707 & 0.693 & 0.721 & $<0.001$ \\
\hline -Years 2015-2018 & 0.582 & 0.569 & 0.594 & $<0.001$ \\
\hline Elixhauser Comorbidity Index & 1.056 & 1.055 & 1.056 & $<0.001$ \\
\hline Rheumatoid Arthritis & 0.859 & 0.810 & 0.911 & $<0.001$ \\
\hline Female & 0.870 & 0.855 & 0.885 & $<0.001$ \\
\hline \multicolumn{5}{|l|}{ Race } \\
\hline -White & Ref & & & \\
\hline -African American & 1.071 & 1.044 & 1.099 & $<0.001$ \\
\hline -Hispanic & 1.144 & 1.108 & 1.180 & $<0.001$ \\
\hline -Asian/Pacific Islander & 1.267 & 1.200 & 1.337 & $<0.001$ \\
\hline -Native American & 1.022 & 0.910 & 1.147 & 0.716 \\
\hline -Other races & 1.263 & 1.201 & 1.329 & $<0.001$ \\
\hline
\end{tabular}

$\mathrm{aOR}=$ Adjusted Odds Ratio; C.I.= confidence interval

Conclusion: In this nationwide study, among hospitalized patients with CDI, those with comorbid RA had significantly decreased odds of inpatient mortality than those without RA. Further studies are needed to understand this association.

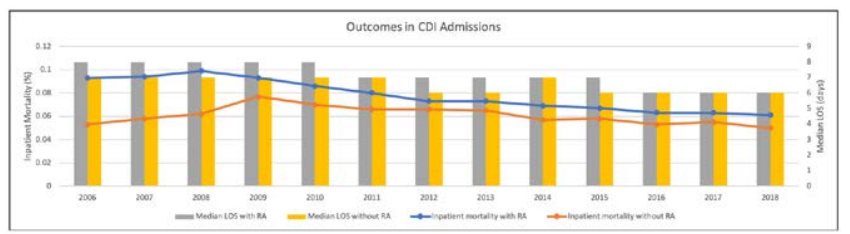

Figure 1.

Disclosure of Interests: None declared DOI: 10.1136/annrheumdis-2021-eular.2176

\section{POS0538 RELATIONSHIP BETWEEN HINDFOOT DEFORMITIES AND ULTRASOUND TENOSYNOVITIS IN RHEUMATOID ARTHRITIS}

K. Maatallah ${ }^{1}$, H. Boussaa ${ }^{1}$, H. Riahi ${ }^{2}$, H. Ferjani ${ }^{1}$, M. Habechi ${ }^{1}$, W. Triki ${ }^{1}$, D. Ben Nessib ${ }^{1}$, M. Bouaziz ${ }^{2}$, D. Kaffel ${ }^{1}$, W. Hamdi'. ${ }^{1}$ Mohamed Kassab Institute of Orthopedics, Rheumatology, La Manouba, Tunisia; ${ }^{2}$ Mohamed Kassab Institute of Orthopedics, Radiology, La Manouba, Tunisia

Background: Rheumatoid arthritis (RA) is an inflammatory disease that frequently affects the joints and soft tissues of the feet. Tibialis posterior tenosynovitis has a reported prevalence between 13 and $64 \%$ in RA. The condition is associated with a progressive flat foot deformity and significant gait disability. However, few studies have investigated the relationship between foot deformities and the involvement of tendons. Recently, ultrasonography (US) has been reported as the gold standard for the investigation of tendons.

Objectives: This study aimed to assess the relationship between hindfoot deformities and US tenosynovitis in RA.

Methods: We conducted a cross-sectional study including patients with RA (ACR/EULAR 2010). Demographic data and disease parameters were collected. For each patient, a podoscope examination of both feet was performed by a rheumatologist. A radiologist experienced in musculoskeletal imaging performed a US examination using a Philips HD11 device with a high-frequency linear transducer. The assessed lesions were synovitis of the tibiotalar, talonavicular, and subtalar joints, and tenosynovitis of tibialis anterior (TA), extensor hallucis longus $(E H L)$, extensor digitorum longus (EDL), tibialis posterior (TP), flexor digitorum longus (FDL), flexor hallucis longus (FHL), fibularis brevis (FB) and fibularis longus (FL) tendons. The presence or absence of synovitis and tenosynovitis was recorded, and the composite synovitis score (power doppler/grayscale ultrasound (PDUS)) was measured for each joint. The US score of each patient was defined by the sum of the composite scores of the joints studied (0-30). A p-value $<0.05$ was considered significant

Results: Sixty-two feet were examined in 31 RA patients ( 25 women and six men) with a mean age of $54.8 \pm 10.8$ years old [32-70]. The mean disease duration was $8.5 \pm 7.2$ years [1-37]. Rheumatoid Factor (RF) and Anti-Citrullinated Peptides Antibodies (ACPA) were positive in respectively $61.3 \%$ and $83.8 \%$ of cases. The mean DAS28 ESR was $3.8 \pm 1.5[0.6-7]$.

Podoscope examination revealed pes planus valgus (PPV) in $55.6 \%$ of cases and pes cavus varus (PCV) in $18.5 \%$ of cases. US showed tibiotalar synovitis in $59.3 \%$ of cases, talonavicular synovitis in $64.8 \%$ of cases, and subtalar synovitis in $46.3 \%$ of cases. In the anterior compartment, tenosynovitis of TA was noted in $5.6 \%$ of cases, of EHL in $1.9 \%$ of cases, and EDL in $9.3 \%$ of cases. In the medial compartment, tenosynovitis of TP was found in $22.2 \%$ of cases, of FDL in $5.6 \%$ of cases, and $\mathrm{FHL}$ in $0 \%$ of cases. In the lateral compartment, tenosynovitis of $\mathrm{FB}$ and $\mathrm{FL}$ was found in $25 \%$ and $11.1 \%$ of cases respectively.

An association between PPV and synovitis of the tibiotalar joint $(p<0.001)$ and the subtalar joint $(p=0.007)$ was found. An association was also noted with $F L$ tenosynovitis $(p=0.045)$ but not with the other assessed tendons.

No association was noted between PCV and synovitis or tenosynovitis of the assessed structures.

Conclusion: PPV was frequent among RA patients. This condition was associated with tibiotalar and subtalar synovitis and FL tenosynovitis. It is important to detect and correct foot deformities in order to ensure optimal control of the disease.

Disclosure of Interests: None declared

DOI: 10.1136/annrheumdis-2021-eular.2223

POS0539

PAIN IS AN INDEPENDENT ASSOCIATED FACTOR ON FUNCTIONAL LIMITATION IN CHINESE PATIENTS WITH EARLY RHEUMATOID ARTHRITIS

Y. W. Zou' ${ }^{1}$, C. Chen ${ }^{1}$, Q. Zhang ${ }^{1}$, S. Y. Lian ${ }^{1}$, K. M. Yang ${ }^{1}$, Y. H. Xu ${ }^{1}$, J. Lin ${ }^{1}$, J. D. Ma ${ }^{1}$, Y. Y. Zou', L. Dai ${ }^{1}{ }^{1}$ Sun Yat-Sen Memorial Hospital, Sun Yat-Sen University, Department of Rheumatology, Guangzhou, China

Background: Rheumatoid arthritis (RA) is a leading cause of extremity disability in Chinese female population according to 2006 national survey. However, less epidemiological data about functional limitation in Chinese RA patients have been published.

Objectives: To investigate the prevalence, characteristics and associated factors of functional limitation in Chinese RA patients.

Methods: Consecutive patients with RA were recruited. The demographic and clinical data were collected including indicators of disease activity, functional assessment and radiographic assessment. According to Health Assessment 
Questionnaire Disease Index (HAQ-DI), functional limitation was defined as: mild $(0<\mathrm{HAQ}-\mathrm{DI} \leq 1)$, moderate $(1<\mathrm{HAQ}-\mathrm{DI} \leq 2)$, and severe $(2<\mathrm{HAQ}-\mathrm{DI} \leq 3)$. Results: There were 643 RA patients recruited with $82.3 \%$ female and mean age $49.7 \pm 12.9$ years. The median (IQR) of total HAQ-DI was $0.25(0.00-0.75)$ and there were $399(62.1 \%)$ RA patients with functional limitation including 293 (45.6\%), 73 (11.4\%), 33 (5.1\%) with mild, moderate, and severe functional limitation, respectively. The highest prevalence of functional limitation subdimension was "walking" (43.5\%), followed by "grip" (36.1\%), "reach" (35.5\%), "common daily activities" (33.4\%), "hygiene" (33.0\%), "dressing and grooming" (29.7\%), "arising" (29.1\%), while the lowest was "eating" (18.4\%). Further age stratification showed that the prevalence of functional limitation was increased with age $(P<0.001)$, but no difference between male and female RA patients (58.8\% vs. $62.8 \%, P=0.426)$. The prevalence of functional limitation of RA patients with disease duration $<1$ years (early), 1-10 years (intermediate) and $\geq 10$ years (long) were $70.2 \%, 55.9 \%$ and $74.5 \%$ respectively, showing a $\mathrm{U}$-shaped curve (Figure $1 \mathrm{~A}$ ), which indicated that early RA also had high rate of functional limitation. Furthermore, early RA patients had the highest proportion of severe functional limitation $(14.3 \%$ vs. $2.0 \%$ vs. $8.7 \%$, Figure $1 \mathrm{~B})$, together with higher prevalence of functional limitation of all eight subdimension than those with intermediate disease duration $(P<0.05$, Figure $1 \mathrm{C})$. There were significant differences in Pain VAS, indicators of disease activity, functional and radiographic assessment among RA patients with different disease duration. Compared with those with intermediate disease duration, early RA patients had higher Pain VAS, higher disease activity indicator (including ESR, CRP, CDAI), higher HAQ-DI, but lower radiographic indicators (all $P<0.05$ ). There was no significant difference in disease activity and functional indicators between early RA patients and those with long disease duration. Multivariate ordered logistic regression analysis showed that Pain VAS $(O R=2.116,95 \% \mathrm{Cl}: 1.483-3.019)$, disease activity indicators [including CRP $(O R=1.047,95 \% \mathrm{Cl}: 1.011-1.084)$ and CDAI $(O R=1.128,95 \% C l: 1.054-1.208)]$ were associated factors of functional limitation in early RA patients.
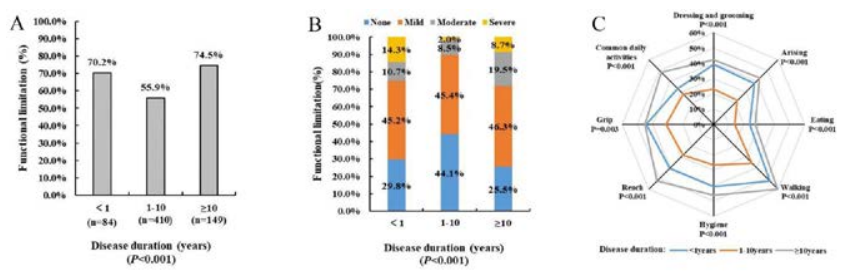

Figure 1. The prevalence of function limitation in RA patients. (A) The prevalence of functional limitation in different disease duration groups of RA patients. (B) The different degrees of functional limitation in different disease duration groups of RA patients. (C) The subdimension characteristics of functional limitation in different disease duration groups of RA patients.

Conclusion: Near two third early RA patients have functional limitation, in which both pain and active disease are independent associated factors. Management of pain and target treatment in early RA patients should be emphasized.

Fund program:

National Natural Science Foundation of China (81801605, 81801606, 81971527); Guangdong Natural Science Foundation (2018A030313541, 2018A030313690, 2019A1515011928); Guangzhou Science and Technology Program (201904010088); Guangdong Basic and Applied Basic Research Foundation (2020A1515110061); Guangdong Medical Scientific Research Foundation (A2018062)

Disclosure of Interests: None declared

DOI: 10.1136/annrheumdis-2021-eular.2291

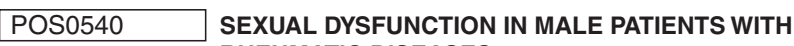 RHEUMATIC DISEASES}

A. P. Stanciu ${ }^{1}$, L. Groseanu ${ }^{1,2}$, A. Balanescu ${ }^{1,2}$, D. Predeteanu ${ }^{1,2}$, D. OprisBelinski $^{1,2}$, V. Bojinca ${ }^{1,2}$, F. Berghea ${ }^{1,2}$, C. L. Constantinescu ${ }^{1,2}$, I. Saulescu ${ }^{1,2}$ S. Daia-lliescu ${ }^{1,2}$, A. Borangiu ${ }^{1,2}$, D. Mazilu ${ }^{1,2}$, M. M. Negru ${ }^{1,2}$, C. Cobilinschi ${ }^{1,2}$, R. Ionescu, ${ }^{1,2}$. ${ }^{1}$ Saint Mary Hospital, Rheumatology and Internal Medicine, București, Romania; ${ }^{2}$ Carol Davila University of Medicine and Pharmacy, Rheumatology, București, Romania

Background: Sexual health is an essential element of overall health and well-being. Rheumatic diseases may affect sexual functioning in many ways related to pain, fatigue, stiffness, functional impairment, depression, anxiety, negative body image, reduced libido, hormonal imbalance and drug treatment. However, these issues are rarely addressed in clinical practice.

Objectives: The aim of this study was to evaluate sexual function in a cohort of men with rheumatic disease compared to healthy controls.
Methods: This was an observational, single-center, cohort study conducted between august 2019 and march 2020 in the Rheumatology department of "Saint Mary" Clinical Hospital in Bucharest which included 120 men with ages between 18 and 60 years - 60 patients with rheumatic diseases and 60 healthy controls. The study tools were the Sexual Health Inventory for Men (SHIM) questionnaire and one questionnaire referring to personal data, history of the rheumatic disease, comorbidities, treatment and sexual impairment. Also, the disease activity was assessed using specific scores for each condition.

Results: In this cohort of 60 patients, the mean age was 45.26 (7.8) years and the diagnoses wereankylosing spondylitis (AS) $-37 \%$,psoriatic arthritis (PsA) - 18\%, rheumatoid arthritis (RA) - 17\%, systemic sclerosis (SS) - $15 \%$ and gout $-13 \%$. More than half of the patients $(62 \%)$ had active disease based on specific scores (ASDAS for AS, DAS28-CRP for RA, EScSG disease activity indices for SS, DAPSA for PSA). Regarding sexual life, this study showed a significant decrease in sexual life quality after rheumatic disease diagnosis(before diagnosis: $71,67 \%$ - satisfying and $16,67 \%$ - not satisfyingversus after diagnosis: $21,67 \%$ - satisfying and $68,33 \%$ - not satisfying). Most patients (90\%) reported impairment of their sexual life after diagnosis. In terms of sexual dysfunction (SD), a significantly higher proportion of patients $(40 \%)$ mentioned reduced libido compared to the control group $(18,33 \%)(p=0.043)$. Also, $21,66 \%$ of the patients reported erectile dysfunction (ED) in comparison with only $8,33 \%$ in the control group $(p=0.009)$. Most patients with AS, RA, PsA and gout had mild ED while most patients with SS presented with mild to moderate ED. Also, the SHIM score mean value was significantly lower in the study group $(17,65)$ compared to the control group $(20,15)(p=0.009)$. The importance of SD in this cohor is emphasized by the fact that only one patient conceived after rheumatic disease diagnosis. Concerning treatment, more than half of the patients $(55 \%)$ reported no effect of the therapy on their sexual life while $38.33 \%$ mentioned that medication improved their sexual life and very few $(7 \%)$ reported a worsening.

Conclusion: This study revealed a higher prevalence of sexual dysfunction in male patients with rheumatic disease in comparison with healthy controls. Considering the importance of sexual and reproductive health rheumatologists should approach this topic with their patients and offer them guidance.

\section{REFERENCES:}

[1] AG Tristano, "The impact of rheumatic diseases on sexual function", Rheumatol Int 2009 Jun;29(8):853-60

Disclosure of Interests: None declared

DOI: 10.1136/annrheumdis-2021-eular.2330

\section{POS0541 LOW PREVALENCE OF SSA (anti-Ro) AND SSB (anti-La) AUTOANTIBODIES IN FEMALE RHEUMATOID ARTHRITIS PATIENTS WITH A WISH TO CONCEIVE}

H. T. Smeele ${ }^{1}$, L. F. Perez-Garcia ${ }^{1}$, J. M. J. Cornette ${ }^{2}$, M. W. Schreurs ${ }^{3}$, R. Dolhain ${ }^{1} .{ }^{1}$ Erasmus MC, Rheumatology, Rotterdam, Netherlands; ${ }^{2}$ Erasmus MC, Department of Gynaecology and Obstetrics, Rotterdam, Netherlands;

${ }^{3}$ Erasmus MC, Department of Immunology, Rotterdam, Netherlands

Background: The presence of SSA (anti-Ro) and SSB (anti-La) autoantibodies has important clinical implications for female patients with a wish to conceive. The association between maternal levels of these autoantibodies and complete congenital heart block and neonatal Lupus syndrome is well established. Currently guidelines advise to test for these antibodies in all Rheumatoid Arthritis (RA) patients with a wish to conceive (1)

Objectives: The objective was to determine the prevalence and titers of SSA and SSB autoantibodies in female patients with rheumatoid arthritis and a wish to conceive or who are pregnant.

Methods: Patients were derived from 2 large prospective cohorts on RA and pregnancy (PARA-cohort and PreCARA-cohort). The presence and titers of SSA and SSB were tested using Phadia, an automated system which uses fluorescence enzyme immunoassays (FEIA) (Thermo-Fisher Scientific).

Results: We included a total of 647 patients with RA and a wish to conceive, $417(64.5 \%)$ conceived during the follow-up period. A detailed description of the study population, stratified for the presence of SSA and SSB antibodies autoantibodies is presented in table 1. A total of 25 out of 647 patients had detectable SSA or SSB antibodies: SSA $n=25$ (SSA-52 $n=17$, SSA-60 $n=19)$, SSB $n=7$. This corresponds with a prevalence of $3.9 \%$ for SSA antibodies and $1.1 \%$ for SSB antibodies. $13(52 \%$ of the SSA positive patients $2.0 \%$ of the total population) patients had a titer of $>240$ units $/ \mathrm{ml}$ of SSA antibodies, and $3(42.9 \%$ of the SSB positive patients, $0.46 \%$ of the total 